\title{
A huggable, mobile robot for developmental disorder interventions in a multi-modal interaction space
}

\author{
Andrea Bonarini ${ }^{1}$, Franca Garzotto ${ }^{2}$, Mirko Gelsomini ${ }^{3}$, \\ Maximiliano Romero ${ }^{4}$, Francesco Clasadonte ${ }^{5}$, Ayşe Naciye Çelebi Yilmaz ${ }^{6}$
}

\begin{abstract}
We propose a new emotional, huggable, mobile, and configurable robot (Teo), which can address some of the still open therapeutic needs in the treatment of Developmental Disability (DD). Teo has been designed in partnership with a team of DD specialists, and it is meant to be used as an efficient and easy-to-use tool for caregivers. Teo is integrated with virtual worlds shown on large displays or projections and with external motion sensing devices to support various forms of full-body interaction and to engage DD persons in a variety of play activities that blend the digital and physical world and can be fully customized by therapists to meet the requirements of each single subject. Exploratory studies have been performed at two rehabilitation centres to investigate the potential of our approach. The positive results of these studies pinpoint that our system endeavors promising opportunities to offer new forms of interventions for DD people.
\end{abstract}

\section{INTRODUCTION}

Developmental Disability (DD) is a broad term encompassing any form of disability that began during the developmental period and is characterized by limitations in intellectual functioning and adaptive behaviour, usually lasting throughout a person's lifetime. Intellectual functioning refers to general mental capacity such as learning, reasoning, problem solving, and imagination. Adaptive behaviour is the collection of conceptual, social, and practical skills that are learned and performed by people in their everyday lives. Deficits in these areas characterize, for instance, persons with Intellectual Disability (ID), Autism Spectrum Disorder (ASD), or cerebral palsy, and have devastating effects on the quality of life of the subject and his/her family.

In the last 20 years, interactive technologies, including robots, have been introduced in the therapeutic practice for DD subjects, in particular ASD children, who seem to respond well. Still, the wide variety of subjects' impairments, and their heterogeneous response to technology enhanced treatment, call for a new gamut of solutions and interaction modalities that are not yet fully explored.

\footnotetext{
${ }^{1}$ Andrea Bonarini is Full Professor at Dipartimento di Elettronica, Informazione e Bioingegneria (DEIB), Politecnico di Milano, Piazza Leonardo da Vinci 32, 20133 Milano, Italy andrea.bonarini@polimi.it

${ }^{2}$ Franca Garzotto is Associate Professor at DEIB

${ }^{4}$ Maximiliano Romero is Assistant Professor at Dipartimento di Design, Politecnico di Milano maximiliano.romero@polimi.it

${ }^{5}$ Francesco Clasadonte is Research Associate at DEIB francesco.clasadonte@polimi.it

${ }^{6}$ Ayşe Naciye Çelebi Yilmaz is PhD Student at DEIB ayse.celebi@polimi.it
}

In this paper, we present Teo, an emotional, huggable, mobile, and configurable robot that exploits, in a novel way, a wide range of integrated interaction modalities and has been designed in cooperation with a team of specialists (psychologists, special educators, and therapists) who have long-term, everyday experience with DD subjects. Teo is a holonomic robot, free of moving on the floor in any direction at a speed comparable to that of people in indoor environments. While maintaining its robotic nature, Teo can be easily perceived as an entity with which it is possible to interact without any specific constraint such as limited speed or bounded kinematics. This stimulates DD subjects to explore the physical space and spatial relationships while interacting with the robot.

Teo can exploit several interaction channels in ways that are completely customizable to the subject's needs and preferences. His soft body includes a set of sensors to detect natural touch such as hugs, slaps, punches, caresses. These sensors do not have the purpose of eliciting any specific response, but are aimed at enabling "natural" tactile interaction and encouraging physical and social relationships with Teo. Delegating these aspects to the robot contributes to progressive release the caregiver's side by side presence. Teo's body displays a set of large buttons positioned on its hat, which support touch-based intentional interaction and can be customized with different labels to enable DD subjects express choice in response to specific requests in different situations.

Coloured lights are hidden in the robots body. They do not appear as an iconic presence but at the same time cannot be ignored when activated. They can be used to strengthen interaction when turned on with a specific color, dynamics and intensity.

Teo can be integrated with an external depth sensor (Kinect) that can sense the motion of human subjects and the robot to enable shared activities that involve full-body interaction in the space. This extended setup includes also a digital display that provides a rich set of multi-media contents and supports a wide range of activities where Teo can play various roles: Feedback (i.e., it acts as a rewarding agent for successful actions performed by the person), Facilitator (i.e., it suggests what to do and when to do it), Prompt (i.e., it acts as a behavior eliciting agent enhancing the entire game play), Emulator (i.e., to promote imitative behavior), Social Mediator (i.e., it encourages social behaviour within the therapist and the DD person, or among peers), and Restrictor (i.e., it constrains the person's movements in the space). 
This paper discusses the design of TEO and its unique set of integrated configurable capabilities. Two exploratory studies are also presented; they have been performed at two different therapeutic centres, with the aim of understanding the potential of TEO to support new innovative forms of intervention for DD subjects.

\section{RELATED WORK}

A number of studies have investigated the application of robots to DD subjects, mostly focusing on ASD children (e.g., [1], [2], [3], [4], [5], [6], [7]), for whom early treatment has been proved particularly effective for the development of basic skills needed for independent living. In most cases, robot shapes and behaviours are developed to support specific learning goals (e.g., imitation or social skills. However, robots have the potential of addressing a wide range of ASD needs; it is acknowledged that when a person establishes a relationship with a robotic agent, it would be important to continue the integration of the robot in the therapy and to adapting it to the new needs arising with the person's development [8].

ASD children are particularly attracted by technological devices, even more if these can move. At the same time, any novelty is a potential source of distress and ASD subjects need to interact with agents that can exhibit expected and predictive behaviors. Due to the difficulty to reliably obtain repeatability of behaviors, most robots used in ASD therapies are remotely controlled. This feature gives the caregivers a complete control of what it is happening during the interaction with the robot, but at the same time may make it clumsy, may introduce delays or undesired reactions in the robot's behavior due to the short time for the operator to decide what should be actuated. This drawback raises the need of another person to complement the therapist's activity, or, if the therapist is alone, creates the extra stress of controlling the robot while also maintaining a relationship with the child. A support in this activity can be done by offering the therapist high level commands that he or she can easily select, leaving to the robot the burden of autonomously implementing detailed actions.

Some researchers suggest that interacting with a robot that is clearly not a person and does not have the behavioral complexity of human beings might be accepted easier by ASD subjects than interacting with a person; hence, it is suggested that the robot can play some of the therapists' roles (e.g., reward, support, stimulus), relieving caregivers from potential problems due to their human-likeness. Many different shapes have been explored for robots used in the ASD domain, from abstract ones to cartoon-like, to simplified humanoids, to realistic human-like faces [2]. Some studies show that it is generally better to avoid the complexity of a human-like face expression, but gaze has an important role in many activities; thus, a completely abstract shape might be confusing for an ASD subject, and the robot's characteristics should provide at least a clear indication of where the robot is looking at.
Very few of the robots used in ASD therapy can successfully exploit movement in space. Most of them are either fixed (e.g., Kaspar [9], Keepon [10]) and somehow manipulable, but not mobile (e.g., Paro [11]). In some cases they have such a slow and clumsy movement (e.g., Nao [12]) that they easily become disturbing or boring, so that children lose attention in movement-related tasks. Notable exceptions are IROMEC [13] and Labo-1 [4], which opened the path toward the exploration of space-related activities involving mobile robots and ASD children. In both cases we have differential drive robots with a rigid shape, running at a speed not comparable with that of a child, so limiting the children's possibility of experiencing the physical space.

Other peculiar characteristics of our approach are the integration of the robot with multimedia contents and virtual worlds presented on projections, and the use of depth sensors (e.g., Kinect) to enable interaction at the distance among the subject, the robot, and the virtual elements on display. Motion-based interaction at the distance has become popular in videogames and has been recently explored in treatments with ASD subjects [14], [15], [16].

Still, its integration with robotic interaction has been seldom investigated. The initial concept and prototype of the system discussed in this paper has been reported in a previous short paper [17].

\section{The DESIGN OF TEO, THE ROBOT}

The design process of Teo's body, behavior, and activities that involve the interaction with it, was performed in partnership with 15 psychologists, therapists and special educators who work daily with DD persons in two different rehabilitation centres. As they didn't have any experience in Robotics, they did not introduce any bias in the design process concerning the possibility of using a robot in their practices: they considered it simply as a potentially useful tool to improve their therapeutic interventions.

The design was informed by the requirements elicited from our observation of regular therapeutic sessions and from focus groups with the specialists to discuss video-recorded therapeutic sessions and the progressive prototypes of the robot. The salient requirements on Teo's body and behaviour which emerged from the elicitation phase are listed in the following paragraphs.

Limited features should be included in the body that recall human shape or face; it should be possible to remove them if a subject would be disturbed by them.

Clean, focused feedbacks should be provided by the robot in response to users' interactions, to support the development of cause-effect understanding.

Teo's behavior and reactions should be consistent with prior - sensory and behavioural - positive experiences of the subjects, to enforce their factual knowledge about the world and to reduce the distress, typical of DD subjects, originated by new unknown phenomena.

The interaction with Teo should be both tangible, i.e., by means of touch and manipulation of the robot, and touchless, 
i.e., at the distance. The former exercises sensorimotor capabilities that, according to embodied cognition theories [18] play a fundamental formative role in the development of different levels of cognitive skills; the latter provides opportunities for exercising awareness and perception of the physical space and its boundaries.

Customizability of Teo's features should be provided, including the physical aspect, interaction capability and behaviours, to adapt the experience with the robot to the specificity of each subject and to have a wide set of possibilities to create new activities with Teo.

It is worth noticing that some of these requirements are not new in robotic research in the ASD arena. The most novel indication emerged from our elicitation process research is the relevance of mixing spontaneous touch-based human-robot interaction with the joint full exploitation of the physical space, which is s largely unexplored requirements dimension.

\section{A. The body}

Teo's final body consists of a robust, triangular, omnidirectional base, only $10 \mathrm{~cm}$ high (diameter $40 \mathrm{~cm}$ ), where motors, batteries, electronics, a coloured LED stripe, infrared and sonar distance sensors are embedded. This gives Teo the possibility to move without limitations on the floor. It implements a human-like kind of kinematics, with unconstrained movement (holonomic) and without the limitations in speed, dexterity, and robustness intrinsic in current stateof-art humanoids. These features make Teo appropriate to engage DD persons in activities that involve the exploration of the physical space and the experience of spatial relationships.

On top of Teo's base there is a soft body made by polystyrene micro balls enclosed in a cloth sack. The tactile sensory experience with this soft, but consistent body is similar to the one with pet toys (plush). A set of $400 \mathrm{~mm}$ long, single-zone Force Sensing Resistors (FSR) optimized for use in human touch control is embedded in the skin, to make Teo able to sense and distinguish hugs, caresses, punches, and, in general, deformations of the body. A Bluetooth speaker is also embedded in the body to provide a mean to emit sounds or vocal output.

Teo's shape - neutral and simple (a kind of ovoid, see Figure 1) - allows for different characterizations, as a head, a cartoon, a body, or just an object. The color - light pastel yellow - is pleasurable, familiar (it can be found in many everyday life contexts), and attractive without being too strong to become disturbing. In its first version Teo had removable arms, which were omitted in the successive prototypes as, following therapists' suggestions, Teo's body should have no relation with any real body that can be found in nature. A simple ovoid yellow body may trigger a mental and emotional association with characters of popular cartoons (e.g., Minions or Barbapapa.) and in any case is intended to generate a feeling of familiarity and safety, without demanding an extensive scrutiny for understanding life-like capability.
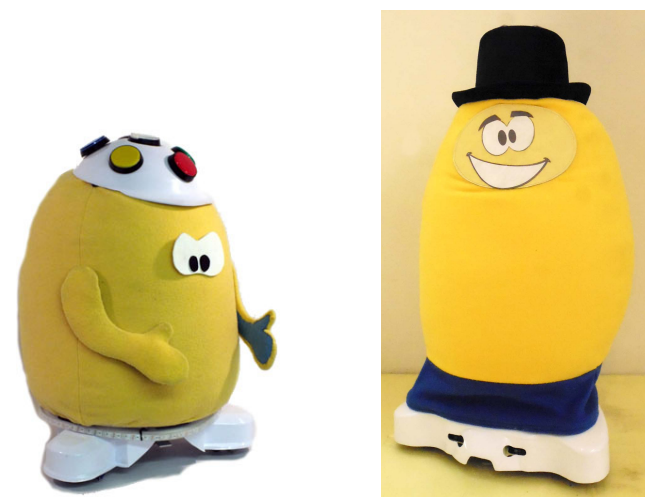

Fig. 1. A first version of Teo, smaller, with cap and velcro-attached eyes, and the final one, with hat and magnetic pad.

Inspired by Mr.Face (a character commonly used in DD therapy), a sheet of magnetic material is placed on the opposite side of the sensible skin. On the magnetic sheet, which has a neutral color and is smoothly integrated in Teo's body, it is possible to attach the components of a face (like eyes, eyelids, or mouths). In this way DD subjects can create their own personalized Teo. This feature is intended to provide a means to familiarize and relax with the robot while also engage in activities related to emotion expression and recognition that are important for ASD subjects. The subjects who are afraid of face expressions may not use this feature, or use it together with their therapists, or experience a face created ad hoc for them.

On the top of the body, a hat can be fixed to hold a set of big push-buttons that can be used for specific interaction activities as described below. The hat can have both a quite recognizable hat shape (like a cylinder hat), which may rise expectations about having also a face on the body, or a more neutral hemispheric cap shape, thus leaving the subject free to interpret it as a hat (possibly making a face below it) or just as a component of a neutral body.

The buttons can be personalized by inserting either colored tags, PCSs (Picture Communication Symbols commonly used in ASD therapy [19]), or iconic images. Buttons are meant to enable children to express choices: in several games, subjects are requested to press the proper button in response to requests from the screen or the robot. For instance, they can be used in task devoted to stimulate associative memory or recognition, as described in Section III-B.

The whole Teo is about $60 \mathrm{~cm}$ high, a size that makes subjects (from 3 years old, up to adults) confident to control it. At the same time, it can easily attract the attention and cannot be left apart when on stage.

\section{B. Interaction design}

The user experience with Teo involves various forms of full-body interaction. In robot manipulation, the subject has a physical contact with Teo, which triggers robot movements or activation of light, sound, vibration effects on Teo's body. In robot-user interaction at a distance, both the subject and the robot can move in the space, and subject's movements 
or position trigger effects similar to those above mentioned. In Joint (robot + subject) interaction at a distance, the robot and the subject interact together with multimedia contents on display, and trigger responses in the virtual world based on the robot's and the subject's position or movements, or the subject's mid-air gestures. A set of games have been developed that exploit this interaction mode, as described in the following.

On screen multimedia contents are designed to provide rewards to the subject and feedbacks to the subject's and the robot's behaviours, to suggest the tasks to be performed in the physical world, or to create simple virtual reality effects. Contents range from simple coloured shapes, animations, and videos, to more sophisticated $2 \mathrm{D}$ and $3 \mathrm{D}$ virtual environments [20]. Users and the robot can see themselves inside the digital world, where they appear as avatars rendered using body silhouettes, mirrored images, or fictitious characters, depending on the game.

Teo can work in three modalities: remotely controlled, autonomously reactive, or autonomously programmed.

As a remotely controlled robot, the therapist can use a device that can be easily hidden in a hand (a joy stick or a blue-tooth pen) to control Teo's behavior. He or she can drive Teo's movements forward and backward, rotate it, or trigger high-level behaviors on Teo's body. The possibility to switch the remotely control mode on-off is available at any time, which enables caregiver to create the best combination of stimuli for a specific subject, to reduce the multi-sensory overload that may arise during the experience with Teo or to make Teo's reactions more evident. In this interaction mode, caregivers can also simulate an additional form of interaction, selecting a phrase from a built-in set or editing a text on the fly, both automatically translated to a voice utterance using a text-to-speech synthesizer. Finally, the remotely controlled mode makes it possible to set up experiences that enlarge the spectrum of emotional engagement and social interaction opportunities: the therapists may decide to have very limited personal, direct interaction with the subjects, and let them experiment forms of "peer-to-peer" socialization with the robot, building social relationships that could hardly be achieved with therapists due to their role of caregivers.

As an autonomously reactive robot, Teo can show reactions to sensor data. In particular, the following behaviours have been implemented.

- Waiting: when it has to wait for someone to interact with, Teo looks around on place, while a part of the LED stripe gets blue;

- Invitation to interact: as soon as someone gets close to Teo, the robot rotates himself towards the subject and verbally invites the person to play;

- Happy: when its body is softly caressed or touched, Teo is pleased and replies by vibrating, rotating itself cheerfully, and moving around, while a green coloured light LED strip blinks slowly;

- Angry: if a person slaps Teo with moderate force, the robot becomes angry and moves sharply towards the person, turning on all the LEDs in red);
- Scared: as soon as someone brutally hits it, Teo becomes timorous and slowly retreats itself, while LEDs become yellowish and pulse slowly.

As an autonomously programmed robot, Teo works autonomously as a play companion and behaves according to its role in the current game. Depending on the game logic and state and on the movements or positions of Teo and the user, an external system drives Teo to specific areas, or activates light, audio, or vibration effects on its body.

The affordances and behaviors implemented in Teo, the integration with external multimedia contents, and the possibility of sensing Teo's and humans' movements and position offer opportunities to engage children in a multiplicity of activities to meet different goals.

"Familiarization" activities are devoted to build a feeling of safety and to reach a state of relaxation, which, for DD subjects, are a precondition for effective participation in any operational or cognitive task. Teo's behaviour and multimedia contents are fully under the remote control of therapists, to help subjects to become acquainted with the new play space, to get to know Teo's affordances and the multimedia characters on display. Examples of familiarization activities are the following. Teo keeps steady, the person sticks face expressions on it, and then light, sound effects, or robot movements are triggered by the therapist consistently with the shaped expression. If the subject speaks to Teo, an appropriate sound is activated in answer. When the subject moves around in the space, Teo follows her. When the subject is close to the display, Teo's avatar appears, and suggests gestures and movements that the subject (and Teo) can imitate.

"Learning" activities are devoted to promote, through play, cognitive and social skills. They involve joint (robot + subject) interaction at the distance with multimedia contents on display and are inspired by simple games frequently adopted in therapeutic centres, which propose choice-making and recognition tasks, simple physical tasks, and storytelling. The subjects must express their willingness to play before each learning activity, by performing a specific gesture towards the screen. In addition, before leaving the room at the end of the entire play session, Teo and his avatar thank the person and greet her, who must respond with a similar gesture. These tasks aim at promoting the ability of expressing personal willingness, often repressed in DD subjects, at enforcing learning of social conventions (greeting when on leave), and at smoothing the negative feeling that the departure from the playground may generate.

So far, learning activities are based on three game scenarios. In "Colours" game, the image of a fruit, an animal, or an object appears on the screen and the child must click the button of the corresponding color on Teo's hat (see Figure 2).

In "Witch Says Colours" game, large color images or shapes are stuck on the floor, and Teo and the person must reach the images on the floor suggested from the display by their respective avatars. In "Shape" game, subjects are required to replicate a target shape shown on screen using their body and moving the robot's body so that their virtual 


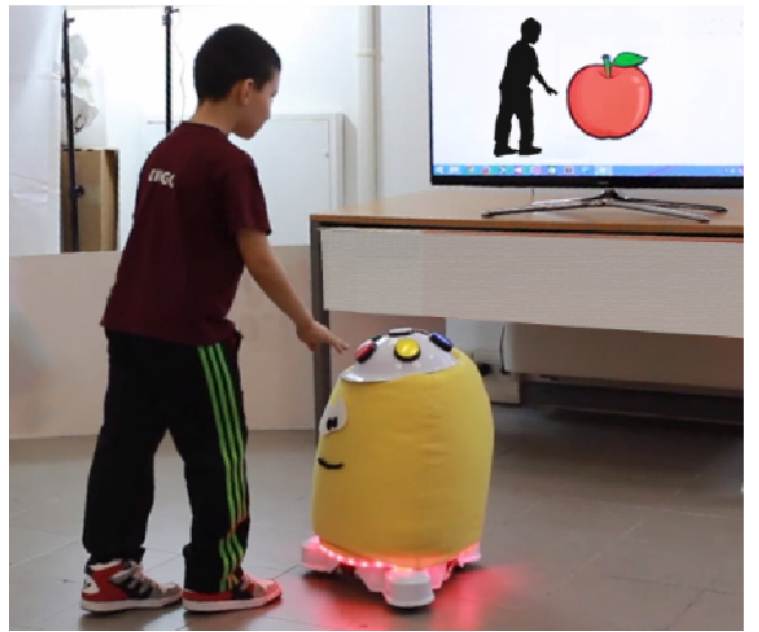

Fig. 2. Playing Color game with Teo.

representation (body silhouettes) appearing on the digital space overlays as much as possible and in the minimal time, the target shape.

All learning activities can be customized by therapists through a simple user-friendly interface, in terms of multimedia contents, virtual characters, rewards, questions, instructions, play time, body movements, level of task complexity, to meet the specific individual needs of each subject at each point of her/his development.

\section{EXPLORATORY STUDIES}

Two exploratory studies have been performed in two therapeutic centres to gain empirical evidence of the potential of our technology as a tool to help DD subjects learn through play with Teo. Overall, the study involved 25 DD subjects and 15 specialists. The total number of sessions with Teo was 88, each one lasting for approximately 15 - 20 minutes. Given the exploratory nature of this reasearch, the different profiles of the involved subjects, and the different therapeutic approaches in the two centres, the two studies share some procedural features, but not a common protocol. In both studies, subjects used our technology as part of their regular treatment. All participants experienced all activities designed for Teo, starting from familiarization tasks (often repeated at the beginning of each session) and proceeding with progressively more complex activities: Colours, Witch Says Colours, and Shape. At least one therapist supervised the subject(s) intervening when needed, while two members of the technical team participated as non-intrusive observers. A dedicated room was instrumented for the purposes of the experimentation. All sessions were video-recorded, using two cameras on the walls to capture simultaneously the subjects' movements from a front view and the multimedia contents on display. A textual report was produced by the therapist at the end of each session. Three focus groups were organized during the study (before, in the middle, and at the end of the experimentation period) involving the therapeutic team and the design and technology team. A meeting with the families of DD participants was organized and all needed authorizations were obtained.

The two studies differ in the detailed plan and execution of the activities, which were defined autonomously by the therapeutic team of each center. They customized the technology and the procedure according to the needs of their subjects and their own therapeutic approaches, and produced textual reports according to their current practice.

\section{A. Exploratory study 1}

1) Participants and procedure: The participants involved in our first study were 19 children (13 males, 6 females) aged 6-12 and 11 therapists or special educators at the rehabilitation center L'Abilità in Milan, Italy, specialized on DD children. Participants attend the centre full time, on a daily base, and their disability include Down Syndrome, Intellectual Disability, autism, Prader-Willy Syndrome and Psychosis. 9 children were profiled as low-functioning, 8 as medium-functioning, and 2 as high functioning.

Children were split in two groups: those with most severe cognitive deficits played alone while children whose socialization problems were more severe than cognitive deficits played with a peer. Each child attended two experimental sessions that took place in two subsequent weeks. In the third week, each child also attended a "group session" with Teo together with his/her classmates (each class is composed of 4-5 subjects with comparable levels of cognitive impairments), performing group activities such as singing with the robot, adding stickers in turn, passing the robot from one child to another.

2) Main Results: The analysis of the data derived from video coding and from the analysis of therapists' and observer's reports was performed by the therapeutic team. It focuses on behavioural variables that concern the emotional, functional, and social sphere. Some variables have been decomposed in sub-variables that are associated with "signals". Signals are observable phenomena (gestures, movements, verbalization, or body expressions) that are interpreted as externalizing feelings, attitudes, needs, or behaviours and are defined in the video coding schema. Behavioural variables are measured as the mean of the number of signals associated to that variable that are detected in a minute. Figure 3 shows the values for the positive behaviour frequency in the two groups (children playing alone, or in pair) and in the two sessions.

Positive behaviour is a macro variable that aggregates all variables denoting improvements in social, functional, or emotional behaviour. For both groups, we notice the increase of positive behaviour expression from session 1 to session 2 in all participants: $+44.84 \%$ in single-player sessions and $+63.76 \%$ in paired sessions. These results indicate that our technology might be beneficial regardless the cognitive level and the play modality. These data also suggest that "playing together" with our technology can more effectively trigger positive behaviours than playing alone. Still, we must consider that the capability of participants assigned to the two modalities differ: in-pair sessions involved higher functioning 


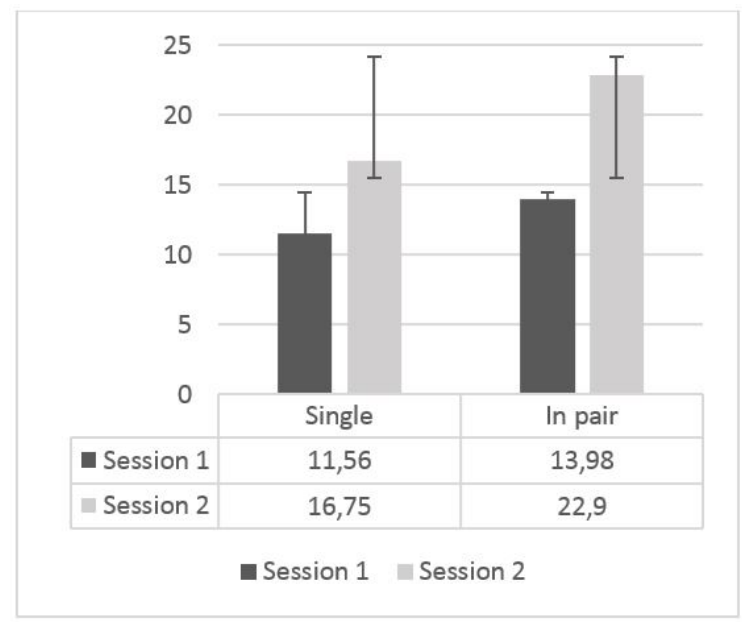

Fig. 3. Mean number of positive behaviour expressions in each of the 2 sessions.

children, which may explain the stronger improvement in this group.

Because of the individual differences among children, even with comparable functioning levels, the data concerning each behavioural variable should be analysed and discussed subject by subject, taking into account the individual characteristics. Figures 4 exemplify the results for one child playing alone, and for another child playing in pair.
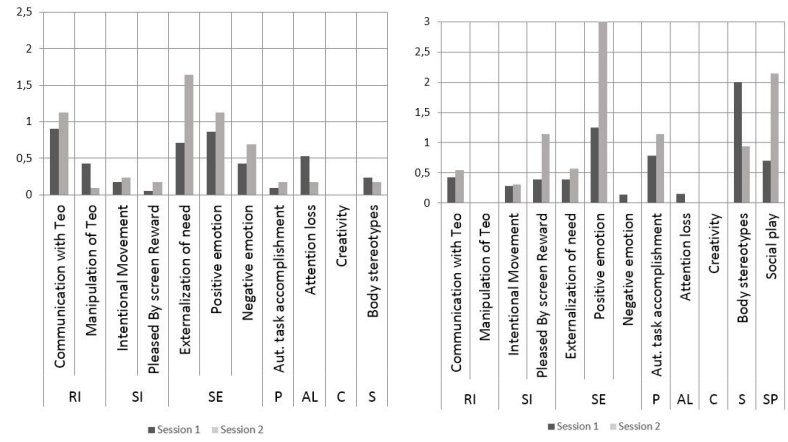

Fig. 4. Comparison of behavioural variables between the 2 sessions in a child playing alone (left) and for a different child playing in pair (right). Legend: RI: Robot Interaction; SI: Screen Interaction; SE: Self Expression P: Performance AL: Attention Loss C: Creativity SP: Social Play.

For lack of space, we summarize here the key findings, by comparing the observations in the first and the second session. Concerning Robot Interaction (RI), manifested by verbal or gestural attempts to communicate with Teo and manipulate him, we detected an increase in communication in the second session for children playing alone. A child even defined a new way to play with the robot: she used one of Teo's accessories, the tie, to engage the robot in a sort of tag game, running away from Teo and using the tie like a matador's cape to attract Teo and to make the robot catch her. This behaviour was considered as a strong manifestation of Creativity (C), which, unfortunately, was not observed in any other participant. In paired-play sessions, we observed increased communication with the robot and decreased manipulation. Still, the overall interaction with the technology increased in session 2: paired children were more attracted by virtual characters, interacted more with the screen, also in comparison with children playing alone. The latter also manifested an increase, but less strong, of Screen Interaction (SI). Self-Expression (SE) capability of both groups of children increased in session 2 .

All children expressed more positive emotions in session 2. Two children playing alone externalized more needs in the second session, and one also manifested more negative emotions. Therapists evaluated both these behaviours as a positive signal, as it denotes increased capability of selfexpression, considering the difficulty of manifesting needs and emotions of any kind that characterize DD subjects. Performance (P), defined by the level of autonomous (aut.) task accomplishment, increased only for 2 children playing alone, and for all children in the other group (who had higher cognitive level). Attention Loss (AL) decreased for all children in session 2, showing that when they get more familiar with technology their attention level improves. Stereotypes (S) in all single-players slightly declined in session 2 (while they were not manifested by higher-functioning subjects in any session). Social Play (SP) skills are considered only for paired play subjects, and showed a progressive increase in all subjects.

\section{B. Exploratory Study 2}

1) Participants and procedure: The second exploratory study was performed at the rehabilitation centre Sacra Famiglia (near Milan), which serves both DD children and DD adults. The study involved less participants ( 5 therapists and 5 low functioning autistic subjects) but for a higher number of sessions (10 per subject). 3 subjects were children ( 2 males, 1 female, aged 3 ) and 2 were adults (males, aged respectively 20 and 25). The sessions were performed on a weekly base, with each subject playing alone under a therapist's supervision.

2) Main Results: Some interesting findings emerged from the data on Task Performance in Color game. We consider, in each session, the number of correct answers to 10 questions of the type "What is the color of this object?" asked by a virtual character on screen, to which subjects respond by pressing a coloured button on Teo's hat (see Fig. 2).

For the two adults we can compare the results of this task with those of an activity aimed at developing the same comptence about "color matching" that the subjects had performed before starting the experimentation with Teo, and using traditional paper-based tools for the same number of sessions.

Figure 5 shows, for each subject, the task results "with" and "without" Teo. The variability in task accomplishment is very high both with and without Teo. Still, we notice higher peaks of task performance when playing with Teo, and a wider difference in the number of correct answers between the first and the last session. 

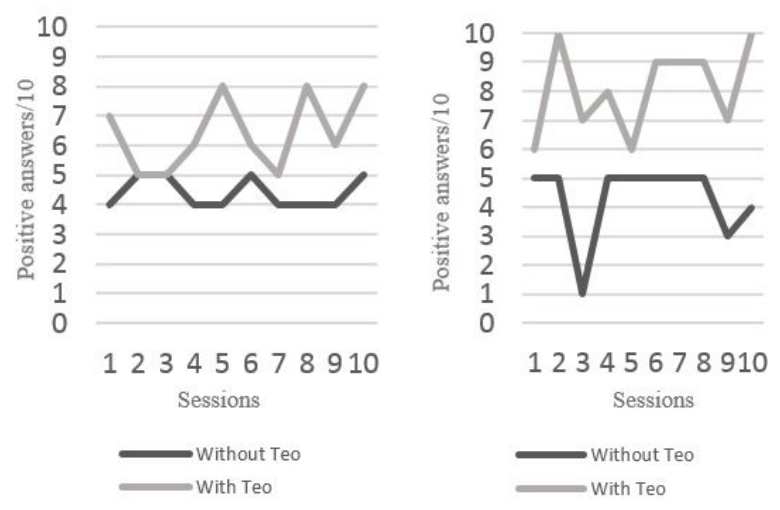

Fig. 5. Adults' performance in Colour game with and without Teo; in abscissas the sessions, in ordinates the number of correct answers to 10 questions.

We cannot provide a similar comparison for the children, as they were novice at the center, and they had not yet performed previous color-matching tasks with conventional materials. Still, comparing the children's number of correct answers in the first and the last session (Figure 6) we may notice an increase of performance. Taking into account that children were not trained to any color matching tasks without Teo before and during the study period, we can ascribe this improvement to the experience done with the robot.

The increase in performance is considered extremely positive by therapists, who claimed that, according to their experience, similar achievements usually require much longer time when using conventional materials.

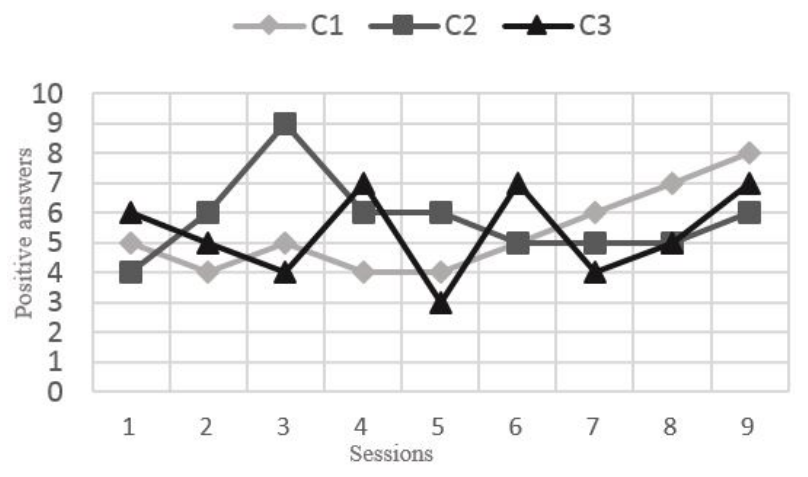

Fig. 6. Children's performance in Color game with Teo; in abscissas the sessions, in ordinates the number of correct answers to 10 questions.

\section{Discussion}

The findings of our empirical studies have limited statistical validity because of the small number of subjects involved, the variability in the way the technology has been used in the different contexts and with the different subjects, the high heterogeneity of the participants in terms of age and impairments, and the lack of baseline measures. As in most studies with DD subjects, causality relationships cannot be defined, as we could not isolate all variables that may have influenced the improvements process. In particular, we do not claim that the benefits we detected have to be ascribed to the experience with Teo, or to some specific features (e.g., the stimuli offered by the robot, the multimedia contents, the full-body interaction paradigm) or a combination of these or other elements such as individual or contextual factors. In addition, we don't know the degree to which the measured benefits may represent a persistent achievement and whether what we have measured in these specific settings can be translated to other contexts and moments of the participants' life.

In spite of the above limitations, all therapists involved in our studies agree that Teo, both used alone and in combination with full-body interaction with multimedia contents on large displays, has elicited functional performances, social behaviours, and emotional responses that either do not occur using traditional methods or require much more time to be achieved; and this happened in all participants regardless the subjects' strong individual differences in intellectual functioning and adaptive behaviour. In addition, it is worth remembering that there is no such thing as an "average" DD subject. Each DD person manifests unique strengths and skill deficits. Things that can reinforce or reward one individual may be unpleasant for another subject. It is also for this reason that all therapists involved in our studies have found the results surprisingly positive.

A final remark concerns the personalization features of our system, which therapists have particularly appreciated. Beside bringing benefits to DD subjects, the customization mechanisms has triggered, and enabled, a process of technology appropriation. Therapists have been able to use and adapt our technology in unexpected ways, not envisaged by the development team, or even deliberately subverting the designers' intentions. Such appropriation is an important and positive phenomenon, in which our stakeholders (the therapists) have acted as catalysts of innovation, and paves the ground towards new therapeutic solutions we cannot even imagine at the moment.

\section{CONCLUSIONS}

Our robotic system is characterized by a unique blend of features that, as suggested by our exploratory studies, may open new, still unknown directions in the interventions for DD subjects. Some of Teo's features are individually available in some existing robots used with DD subjects: support to both touch and touchless interaction, huggability, mobility, multisensory body effects (light, sound, and vibration). Still, to our knowledge, such features have never been combined and harmonized at the degree offered by Teo, nor have been specifically designed and optimized for DD subjects.

In addition, the integration of TEO and its multisensory effects with multimedia on-screen contents and virtual characters, the full-body interaction mode available to the users, to Teo, and among them, offer to DD subjects the opportunity to blend in a unique way the experience of visual technology with the experience of the physical space and their own body; the whole set-up provides a multiplicity of stimuli 
that are attractive and engaging for the subjects and are fully controllable by therapists, creating a context of predictable, clear, repeatable cause-effect phenomena.

The two centres we are collaborating with have expressed strong interest to continue the experimentation after the preliminary exploratory phase. A systematic study has recently started in the two locations and will last for 6 months. It is involving a larger $(n=42)$ but still weakly homogeneous sample, and is based on a shared protocol of activities, measures, and reporting methods. From a design and technological perspective, we are working to extend both Teo's capability and the multimedia contents to include some verbal dialogue capability in the robot and storytelling features in the on-screen virtual worlds. These improvements will enable the creation of experiences with Teo that mimic realistic "everyday" scenarios, and enable DD subjects to train various skills related to intellectual functioning and adaptive behaviour that are needed for their everyday life.

\section{ACKNOWLEDGMENTS}

The authors warmly thank children, therapists, and managers from L'Abilità and Sacra Famiglia centres.

The KROG project is partially funded by Politecnico di Milano within the Social Responsibility Program "Polisocial" 2014-2015.

\section{REFERENCES}

[1] J. J. Diehl, L. M. Schmitt, M. Villano, and C. R. Crowell, "The clinical use of robots for individuals with autism spectrum disorders: A critical review," Research in autism spectrum disorders, vol. 6, no. 1, pp. 249262, 2012.

[2] F. Michaud, A. Duquette, and I. Nadeau, "Characteristics of mobile robotic toys for children with pervasive developmental disorders," in Systems, Man and Cybernetics, 2003. IEEE International Conference on, vol. 3. IEEE, 2003, pp. 2938-2943.

[3] J.-J. Cabibihan, H. Javed, M. Ang Jr, and S. M. Aljunied, "Why robots? a survey on the roles and benefits of social robots in the therapy of children with autism," International journal of social robotics, vol. 5, no. 4, pp. 593-618, 2013.

[4] K. Dautenhahn, I. Werry, T. Salter, and R. T. Boekhorst, "Towards adaptive autonomous robots in autism therapy: Varieties of interactions," in Computational Intelligence in Robotics and Automation, 2003. Proceedings. 2003 IEEE International Symposium on, vol. 2. IEEE, 2003, pp. 577-582.

[5] W. Den Brok and P. Sterkenburg, "Self-controlled technologies to support skill attainment in persons with an autism spectrum disorder and/or an intellectual disability: a systematic literature review," Disability and Rehabilitation: Assistive Technology, vol. 10, no. 1, pp. 1-10, 2015.

[6] B. Scassellati, H. Admoni, and M. Mataric, "Robots for use in autism research," Annual review of biomedical engineering, vol. 14, pp. 275294, 2012.

[7] D. J. Ricks and M. B. Colton, "Trends and considerations in robotassisted autism therapy," in Robotics and Automation (ICRA), 2010 IEEE International Conference on. IEEE, 2010, pp. 4354-4359.

[8] C. Ferrara and S. D. Hill, "The responsiveness of autistic children to the predictability of social and nonsocial toys," Journal of Autism and Developmental Disorders, vol. 10, no. 1, pp. 51-57, 1980.

[9] B. Robins, K. Dautenhahn, R. Te Boekhorst, and A. Billard, "Robotic assistants in therapy and education of children with autism: can a small humanoid robot help encourage social interaction skills?" Universal Access in the Information Society, vol. 4, no. 2, pp. 105-120, 2005.

[10] H. Kozima, M. P. Michalowski, and C. Nakagawa, "Keepon. a playful robot for research, therapy, and entertainment," International Journal of Social Robotics, vol. 1, no. 1, pp. 3-18, 2009.
[11] P. Marti, A. Pollini, A. Rullo, and T. Shibata, "Engaging with artificial pets," in Proceedings of the 2005 annual conference on European association of cognitive ergonomics. University of Athens, 2005, pp. 99-106.

[12] S. Shamsuddin, H. Yussof, L. Ismail, F. A. Hanapiah, S. Mohamed, H. A. Piah, and N. I. Zahari, "Initial response of autistic children in human-robot interaction therapy with humanoid robot nao," in Signal Processing and its Applications (CSPA), 2012 IEEE 8th International Colloquium on. IEEE, 2012, pp. 188-193.

[13] B. Robins, K. Dautenhahn, E. Ferrari, G. Kronreif, B. Prazak-Aram, P. Marti, I. Iacono, G. J. Gelderblom, T. Bernd, F. Caprino, et al., "Scenarios of robot-assisted play for children with cognitive and physical disabilities," Interaction Studies, vol. 13, no. 2, pp. 189-234, 2012.

[14] L. Bartoli, F. Garzotto, M. Gelsomini, L. Oliveto, and M. Valoriani, "Designing and evaluating touchless playful interaction for asd children," in Proceedings of the 2014 conference on Interaction design and children. ACM, 2014, pp. 17-26.

[15] A. Bhattacharya, M. Gelsomini, P. Pérez-Fuster, G. D. Abowd, and A. Rozga, "Designing motion-based activities to engage students with autism in classroom settings," in Proceedings of the 14th International Conference on Interaction Design and Children. ACM, 2015, pp. 6978.

[16] L. Malinverni, J. Mora-Guiard, V. Padillo, L. Valero, A. Hervás, and N. Pares, "An inclusive design approach for developing video games for children with autism spectrum disorder," Computers in Human Behavior, 2016.

[17] A. Bonarini, F. Garzotto, M. Gelsomini, and M. Valoriani, "Integrating human-robot and motion-based touchless interaction for children with intellectual disability," in Proceedings of the 2014 International Working Conference on Advanced Visual Interfaces. ACM, 2014, pp. 341-342.

[18] M. Wilson, "ix views of embodied cognition." Psychonomic Bulletin and Review, no. 9, pp. 625-636, 2002.

[19] F. Garzotto and M. Bordogna, "Paper-based multimedia interaction as learning tool for disabled children," in Proceedings of the 9th International Conference on Interaction Design and Children (IDC '10). New York, NY, USA: ACM, 2010, pp. 79-88.

[20] F. Garzotto and M. Forfori, "Hyperstories and social interaction in $2 \mathrm{~d}$ and $3 \mathrm{~d}$ edutainment spaces for children," in Proceedings of the seventeenth conference on Hypertext and hypermedia (HYPERTEXT '06). New York, NY, USA: ACM, 2006, pp. 57-68. 